\title{
DESENVOLVIMENTO MORFOLÓGICO E DOS OVÁRIOS DE KALLIAPSEUDES SCHUBARTI MAÑE-GARZON (CRUSTACEA, TANAIDACEA) DO CANAL DE SÃO SEBASTIÃO, SÃO PAULO, BRASIL
}

\author{
Fosca Pedini Pereira Leite ${ }^{1}$ \\ Paulo Eduardo Pereira Leite ${ }^{2}$
}

\begin{abstract}
MORPHOLOGICAL DEVELOPMENT AND OVARY HISTOLOGY OF KALLIAPSEUDES SCHUBARTI MAÑE-Garzon (CRUSTACEA, TANAIDACEA) From São SEBASTIÃo CHANNEL, SÃo PAULO, BraSIL. The morphological stages of the Kalliapseudes schubarti Mañe-Garzon, 1949 females were easily determinated and they were similar, but the male stages show distint forms probably due to the ambient stimuli. The description of the male stages demonstrate that K. gianucai (Bacescu, 1979) is another male stage of $K$. schubarti, probably a secondary copulatory stage. The morphology and development of the ovary is related both with the maturity and with the morphological modifications of females stages.

KEY WORDS. Crustacea, Tanaidacea, morphology, development, stages, ovary
\end{abstract}

A distribuição do tanaidáceo tubícola Kalliapseudes schubarti Mañe-Garzon, 1949 está restrita, até o presente, ao Brasil e Uruguai (LANG 1956). É muito abundante em regiões estuarinas e baixios lodosos do Estado de São Paulo (TOMMASI 1970; LeITE 1995), Paraná (LANA et al. 1989) e Rio Grande do Sul (CAPITOLI et al. 1978; BEMVENUTI 1987).

São conhecidos poucos estudos sobre a fase larval, ciclo de vida e estágios de desenvolvimento marsupial e pós-marsupial de espécies de tanaidáceos (BUCKLE-RAMTREZ 1965; JOHNSON \& ATTRAMADAL 1982; MESSING 1983; MASUNARI 1983; MODLIN \& HARRIS 1989). GARDINER (1975) estudou as modificações das gônadas durante o ciclo de vida através de cortes histológicos.

Apesar da sua grande abundância numérica, atribuída à sua elevada capacidade reprodutiva e ampla tolerância à variações ambientais (LEITE 1995), pouco se conhece sobre a sua biologia, especialmente a relacionada com o crescimento e maturidade.

1) Departamento de Zoologia, Instituto de Biologia, Universidade Estadual de Campinas. Caixa Postal 6109, 13084-970 Campinas, São Paulo, Brasil.

2) Departamento de Histologia e Embriologia, Instituto de Ciências Biológicas, Universidade de São Paulo. Caixa Postal 66208, 05389-970, São Paulo, São Paulo, Brasil. 


\section{MATERIAL E MÉTODOS}

As coletas foram realizadas entre outubro de 1989 a julho de 1993 na região do Araçá, localizada a um quilômetro ao sul de São Sebastião, litoral norte do Estado de São Paulo, na porção central do canal (Fig. 1). A região do Araçá é uma pequena enseada constituída de sedimento fino a muito fino, com a presença de vegetação de mangue e afluxo de água doce de influência antrópica (LEITE 1995).

As amostras de sedimento foram retiradas com auxílio de amostrador quantitativo. Os animais foram separados do sedimento por lavagem sobre peneiras e triados para separação de todos os estágios de desenvolvimento. Os espécimes foram fixados em álcool à $70 \%$. Para classificação dos estágios de desenvolvimento, os apêndices retirados dos animais foram montados em lâminas com glicerina e desenhados.

Os animais foram medidos na região mediana dorsal, desde a extremidade anterior da cabeça até a extremidade posterior do pleotelson, através de projeção com câmara clara e com auxílio de lâmina milimetrada também projetada.

Os animais foram separados por sexo e maturidade, para determinação dos estágios de desenvolvimento, de acordo com MEssing (1983): manca I (sem pereiópodo VII); manca II (com pereiópodo VII mas não segmentado e rudimentar); manca III ou neutrum (com pereiópodo VII completamente desenvolvido); fêmea jovem ou preparatória ou não ovígera (com presença de oostégitos rudimentares); fêmea ovígera (com marsúpio desenvolvido, com ovos ou embriões); macho (com quelípodos alongados e alargados, antenas com o primeiro artículo basal mais longo que o das fêmeas).

Foram realizados cortes histológicos para as análises da morfologia dos ovários de fềmeas jovens e ovígeras. A montagem das lâminas permanentes seguiu rotina histológica em parafina para hematoxilina-eosina (HE) com o material fixado préviamente em álcool a $70 \%$. Foram feitos cortes de $7 \mu \mathrm{m}$ de espessura. As lâminas foram fotografadas em fotomicroscópio Nikon Labophot.

\section{RESULTADOS E DISCUSSÃO}

\section{VARIAÇÃO MORFOLÓGICA DOS ESTÁGIOS DE DESENVOLVIMENTO}

\section{Estágios marsupiais}

O ovo (estágio I) tem forma arredondada e apresenta uma membrana externa (Fig. 2).

O embrião (estágio II) tem forma alongada, mostrando marcada curvatura dorsal com o bordo superior liso. Apresenta segmentos rudimentares no bordo inferior (Fig. 3).

A larva manca I (estágio III) apresenta corpo alongado e estruturas como cabeça, antenas, quelípodos e pereiópodos já diferenciados. Nota-se ausência de pleópodos (Fig. 4). 


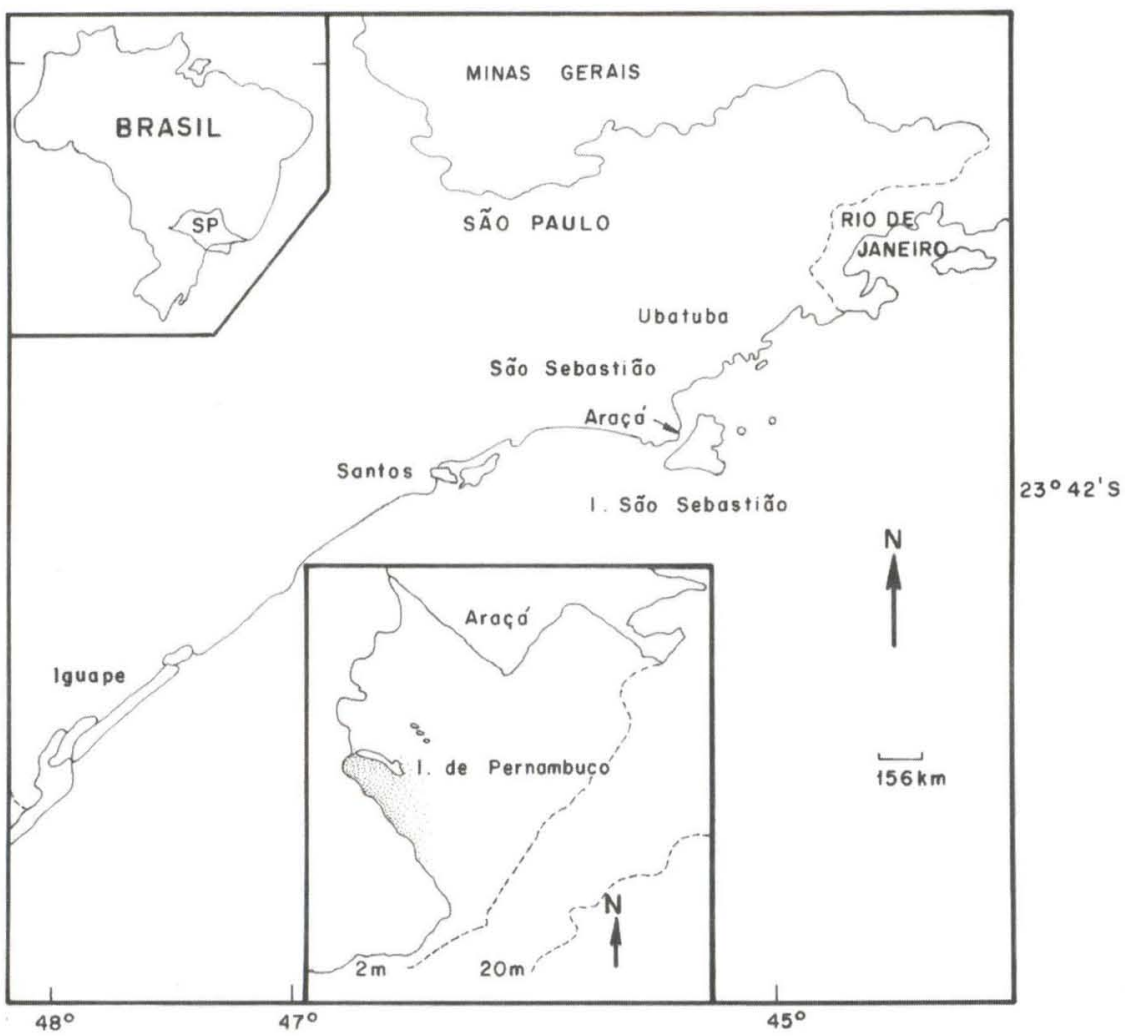

Fig. 1. Região do Araçá, São Sebastiäo, São Paulo. No detalhe a área de estudo.

A larva manca II (estágio IV) apresenta antena 1 com oito artículos sendo o primeiro mais longo e com cerdas escassas. O quarto artículo apresenta um flagelo secundário com uma articulação e sem cerdas (Fig. 5). O quelípodo, com forma semelhante à das fêmeas, apresenta meropodito pequeno e carpopodito longo e ventralmente guarnecido de longas cerdas (Fig. 10).

O desenvolvimento marsupial de K. schubarti transcorre sem mudas como em outras espécies de tanaidáceos (SCHOLL 1963; BUCKLE-RAMIREZ 1965) e semelhante ao descrito por HoLDICH (1968) para uma espécie de isópodo.

A larva manca II deixa o marsúpio para o meio externo. BUCKLE-RAMIREZ (1965) relata que ao estádio de manca II segue-se um estádio juvenil pós-marsupial, denominado manca III ou Neutrum. Os indivíduos deste estádio, após nova muda, se diferenciam sexualmente em machos ou fêmeas jovens.

\section{Estágios pós-marsupiais}

A determinação destes estágios foi baseada no desenvolvimento das antenas 1 e quelípodos, estruturas consideradas caracteres sexuais secundários. Os outros apêndices e maiores detalhes podem ser observados nos trabalhos de LANG (1956). 


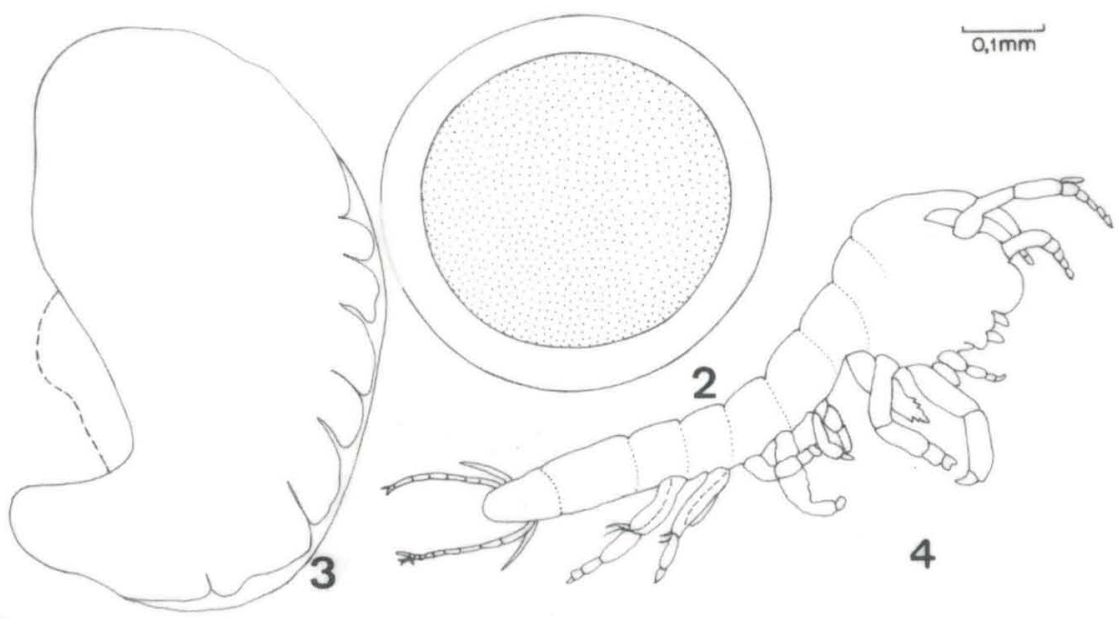

Figs 2-4. Estágios marsupiais. (2) Ovo; (3) embrião; (4) manca I.

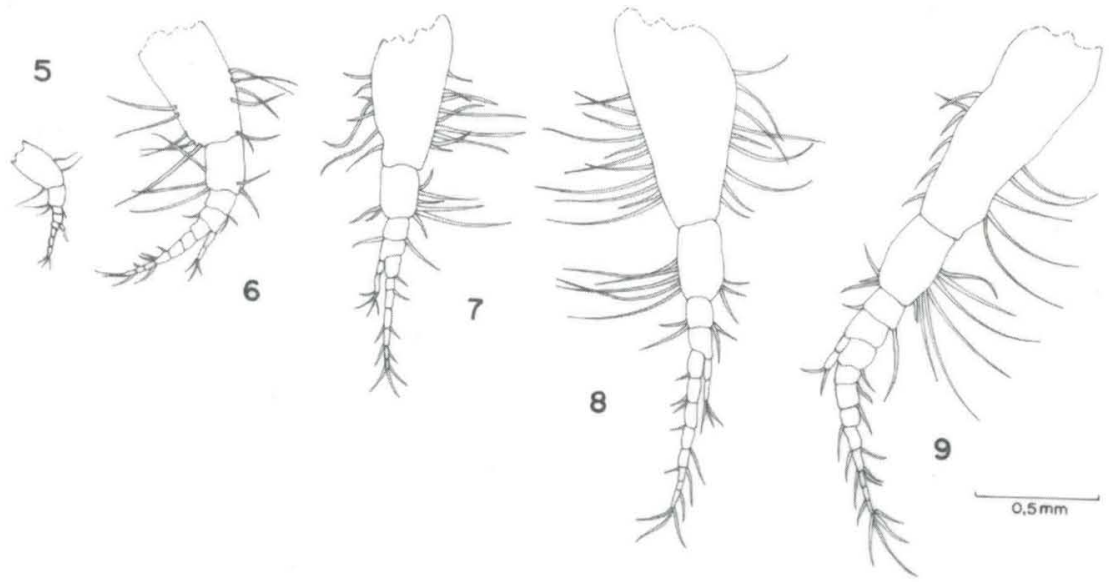

Figs 5-9. Antena 1. (5) Manca II; (6) manca III; (7) fêmea jovem; (8) macho I e II.

\section{Manca III ou Neutrum (estágio V)}

O comprimento total dos indivíduos neste estágio variou entre 5 a $20 \mathrm{~mm}$.

A antena 1 tem 11 artículos sendo o primeiro com maior número de cerdas. O quarto artículo apresenta um flagelo secundário com duas articulações e com cerdas na extremidade distal (Fig. 6).

O quelípodo apresenta meropodito pequeno, carpopodito longo guarnecido de densa fileira de longas cerdas na superfície ventral e apresentando duas cerdas na superfície dorsal. Margem cortante do propodopodito irregularmente serrilhada e o dátilo apresentando na margem interna espinhos voltados para a região anterior (Fig. 11). 


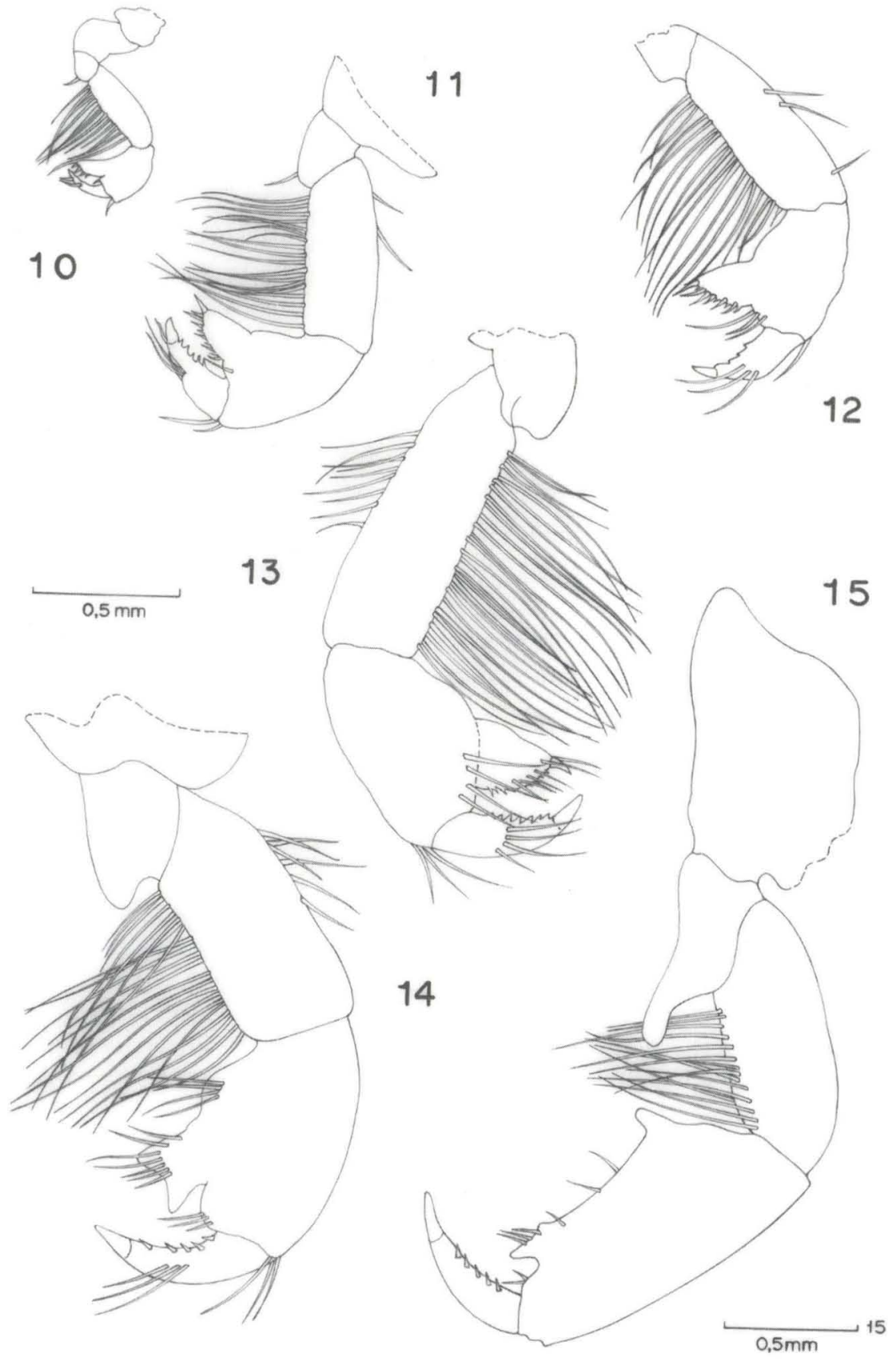

Figs 10-15. Quelipodo. (10) Manca II; (11) manca III; (12) fêmea jovem; (13) fêmea ovigera; (14) macho I; (15) macho II. 


\section{Fêmea jovem (estádio VI)}

Estas fêmeas apresentam comprimento do corpo variando entre 29 a $85 \mathrm{~mm}$.

A antena 1 apresenta 11 artículos sendo o primeiro mais longo e com maior número de cerdas. Há poucas e curtas cerdas na inserção dos artículos do flagelo. $\mathrm{O}$ quarto artículo apresenta um flagelo secundário biarticulado com quatro cerdas apicais (Fig. 7).

O quelípodo tem meropodito pequeno, carpopodito densamente guarnecido na superficie ventral por fileira de longas cerdas e apresentando número escasso (três) de cerdas na superfície dorsal. Margem cortante do propodopodito serrilhada e margem interna do dátilo apresentando espinhos voltados para a região anterior (Fig. 12).

\section{Fêmea ovígera (estádio VII)}

O comprimento total destas fêmeas variou entre 37 a $93 \mathrm{~mm}$.

A antena 1 apresenta 12 artículos. O primeiro, mais longo, está guarnecido de cerdas e o quarto apresenta um flagelo secundário biarticulado com três cerdas apicais (Fig. 8).

O quelípodo tem meropodito comparativamente maior que o das fêmeas nos outros estágios de maturidade. Apresenta carpopodito mais alongado, com grande número de longas cerdas na superfície ventral em fileira, ocorrendo também oito cerdas na superfície dorsal. Margem cortante e dátilo semelhantes aos já descritos para os outros estágios (Fig. 13).

\section{Macho (estádio VIII)}

O comprimento total variou entre 29 a $93 \mathrm{~mm}$.

A antena 1 apresenta 13 artículos, sendo o primeiro mais longo e fino que o das fêmeas (caráter dimórfico) e guarnecido de cerdas. O quarto artículo apresenta um flagelo secundário biarticulado com duas cerdas distais (Fig. 9).

Os machos apresentaram duas formas distintas de quelípodo com variação morfológica acentuada especialmente com relação ao tamanho dos própodito e meropodito.

Uma das formas (macho I), semelhante à descrita por LANG (1956), apresenta o meropodito do quelípodo mais desenvolvido que os das fêmeas, notando-se na região ventral uma protuberância ou apófise desenvolvida; carpopodito alongado mas menor que o das fêmeas, ostentando fileiras de longas cerdas na superfície ventral. O propodopodito apresenta-se mais largo e desenvolvido com protuberância na superfície ventral, margem cortante com duas elevações dentiformes altenado-se com cerdas. Margem interna do dátilo com espinhos (Fig. 14).

A segunda forma (macho II) é semelhante à descrita por BACESCU (1979) para Kalliapseudes gianucai coletada na Lagoa dos Patos (Rio Grande do Sul). Nesta o quelípodo apresenta meropodito com apófise forte e curva, carpopodito semelhante ao da primeira forma (LANG 1956) mas com cerdas mais curtas e menos numerosas, propodopodito com apófise proximal ventral dentiforme e margem cortante com elevações proximas à inserção do dátilo. Margem interna do dátilo com espinhos (Fig. 15). BACESCU (1979) considerou, contudo, que a espécie por ele descrita se parecia, em muitos aspectos, com K. schubarti. 
As duas formas de quelípodo do macho indicam claramente que a descrita por BACESCU (1979) pertence a outra fase de desenvolvimento de K. schubarti e não a uma outra espécie. Estes machos II podem ser originados de fềmeas já presentes na população (HOLDICH \& JONES 1983; GARDINER 1975) causando, desta forma, problemas taxonômicos. SEXTON (1924) apontou que muitas das dificuldades em classificar os crustáceos eram devidas às extraordinarias modificações causadas pelo crescimento e sexo. O não conhecimento sobre os estágios de desenvolvimento leva a muitos erros especialmente para os tanaidáceos (LANG 1956; BUCKLE-RAMIREZ 1965). BUCKLE-RAMIREZ (1965) associou o aparecimento de uma segunda forma de machos às condições ambientais. Anteriormente, LANG (1956) já havia relacionado a protoginia dos tanaidáceos à fatores semelhantes. Provavelmente estas condições estão relacionadas à ocupação de espaço e à estratégia reprodutiva. Um grande número de fềmeas reprodutivas presentes no ambiente aumentaria, desta forma, a chance de estabelecimento, permanência e sucesso da espécie em um determinado local. Os machos não se alimentam, desempenham apenas papel reprodutivo e poderiam quando escassos se desenvolver de fêmeas, para a reprodução (GARDINER 1975).

Os tanaidáceos podem ser hermafroditas potenciais (BUCKLE-RAMIREZ 1965 ) e, simultaneamente, protandricos e protogínicos (CHARNIAUX-CotTON 1960).

Kalliapseudes schubarti, pelo fato de apresentar duas formas distintas de machos, poderia ser um hermafrodita em potencial, mas outros estudos são necessários para esclarecer a presença das formas diferentes. É uma espécie oportunista, presente nas amostras da Região do Araçá em grande número de indivíduos (LEITE 1995) e provavelmente utiliza esta estratégia de ocupação do espaço. Os machos secundários seriam necessários para nova fecundação das fêmeas.

\section{OVÁRIO DAS FÊMEAS}

Os cortes histológicos de fêmeas não ovígeras e ovígeras de Kalliapseudes schubarti mostram as alterações dos ovários entre as duas fases de maturidade.

Os ovários de fêmea imatura apresentam ovócitos já completamente formados (Fig. 16), enquanto que os de uma fềmea ovígera (Fig. 17) não são facilmente visiveis, por estarem vazios pois os ovócitos foram liberados para o marsúpio. A figura 18 mostra ovos dentro do marsúpio de uma fêmea com o ovário vazio.

Fêmeas jovens, também chamadas de preparatórias, mostram aumento do desenvolvimento dos ovários e dos ovócitos (BUCKLE-RAMIREZ 1965), isto é, com aumento de vitelo preenchendo os ovários. Ovócitos desenvolvidos (OV) de $K$. schubarti podem ser vistos na figura 16 , notando-se o aspecto granular proporcionado pelo vitelo. Mais internamente percebe-se a presença de núcleo e citoplasma.

Após os ovos serem liberados no marsúpio, os ovários contraem-se tanto em diâmetro com em extensão e, geralmente, não são visualizados facilmente (BUCKLERAMIREZ 1965). O mesmo aspecto pode ser notado nas fêmeas ovígeras de $K$. schubarti (Fig. 17) que apresenta paredes colapsadas sem a presença de ovócitos provavelmente devidas ao esvaziamento total dos ovários. Este ciclo de desenvolvimento dos ovos presumivelmente poderá ser repetido, com a nova produção de 
ovos durante um segundo estágio copulatório das fêmeas (GARDINER 1975). LEITE (em preparacão) notou duas ou mais modas de distribuição dos comprimentos das fêmeas ovígeras em alguns meses indicando a possibilidade de reprodução contínua. Por outro lado o desvio da razão sexual em favor das fêmeas (LEITE, em preparacão) pode estimular e indicar a possibilidade de surgimento de machos II na população.

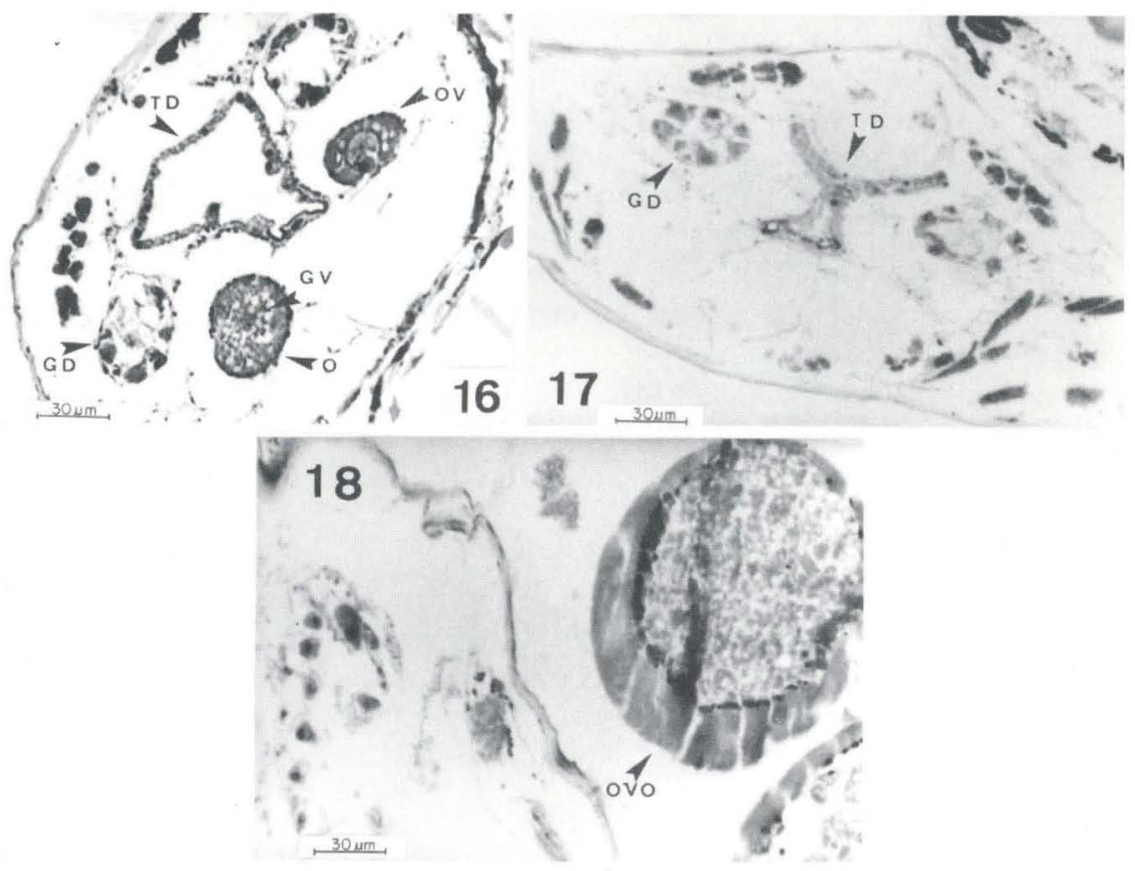

Figs 16-18. Cortes histológicos. (16) Fêmea jovem; (17) fêmea ovigera; (18) fêmea ovigera com ovos no marsúpio. (O) Ovário; (OV) ovócito; (GV) grânulo de vitelo; (GD) glândula digestiva; (OVO) ovo no marsúpio; (TD) tubo digestivo.

AGRADECIMENTOS. À Fundação de Amparo à Pesquisa do Estado de São Paulo, FAPESP (Proc. 89/0995-1), ao Conselho Nacional de Desenvolvimento Científico e Tecnológico, CNPq (Proc. 401300/89-7) pelos Auxílios à Pesquisa e ao Centro de Biologia Marinha da Universidade de São Paulo, CEBIMar pelo apoio logístico.

\section{REFERÊNCIAS BIBLIOGRÁFICAS}

BACESCU, M. 1979. Kalliapseudes gianucai a new Tanaidacea from the brazilian waters. Rev. Roum. Biol. Biol. Anim. 24 (1): 3-8.

BUCKLE-RAMIREZ, L.F. 1965. Untersuchungen über die Biologie von Heterotanais oerstendi Kroyer (Crustacea, Tanaidacea). Z. Morph. Ökol. Tiere 55: 714-782. 
BEMVENUTI, C.E. 1987. Predation effects on a benthic community in estuarine soft sediments. Atlântica, Rio Grande, 9 (1): 3-8.

Capitoli, R.R.; C.E. Bemvenuti \& N.M. GianuCA. 1978. Estudo de ecologia bentônica na região estuarial da Lagoa dos Patos.I. As comunidades bentônicas. Atlântica, Rio Grande, 3: 5-22.

CharniauX-Cotton, H. 1960. Sex determination, p.411-447. In: T.H. WaterMAN (Ed.). The Physiology of Crustacea. New York, Academic Press, 560p.

GARDINER, L.F. 1975. The systematics, postmarsupial development and ecology of the deep-sea family Neotanidae (Crustacea: Tanaidacea). Smithson. Contr. Zool. 170: 1-275.

HoLDICH, D.M. 1968. Reproduction, growth and bionomics of Dynamene bidentata (Crustacea: Isopoda). Jour. Zool. Lond. 156: 137-153.

Holdich, D.M. \& J.A. JonES. 1983. Tanaids. Synopsis of British fauna, new series: 27. Cambridge, Cambridge University Press, 98p.

JoHNSON, S.B. \& T.G. ATTRAMADAL. 1982. Reprodutive behavior and larva developmment of Tanais cavolinii (Crustacea: Tanaidacea). Mar. Biol. 71: 11-16.

Lana, P.C.; M.V.O. Almeida; C.A.F. Freitas; E.C.G. Couto; L.M.P. ContI; A.L. Gonzales-Peronti; A.G. Giles; M.J.S. Lopes; M.H.C. Silva \& L.A. PEDROSO. 1989. Estrutura espacial de associações macrobênticas sublitorais da gamboa Perequê (Pontal do Sul, Paraná). Neritica 4 (1/2): 119-136.

LANG, K. 1956. Tanaidacea aus Brasilien. Kieler Meereesf. 12 (2): 249-260.

LEITE, F.P.P. 1995. Distribuição temporal e espacial de Kalliapseudes schubarti Mañe-Garzon, 1949 (Tanaidacea, Crustacea) da região do Araçá, São Sebastião (SP). Arq. Biol. Tecnol. 38 (2): 605-618.

MASUNARI, S. 1983. Postmarsupial development and population dynamics of Leptochelia savigny (Kroyer,1842) (Tanaidacea). Crustaceana 44 (2): 152162.

Messing, C.G. 1983. Postmarsupial development and growth of Pagurapseudes largoensis McSweeny (Crustacea, Tanaidacea). Jour. Crust. Biol. 3 (3): 380408.

MODLIN, R.F. \& P.A. HARRIS. 1989. Observations on the natural history and experiments on the reproductive strategy of Hargeria rapax (Tanaidacea). Jour. Crust. Biol. 9 (4): 578-586.

SEXTON, E.W. 1924. The moulting and growth-stages of Gammarus, with descriptions of normals and intersexes of $G$. chevreuxi. Jour. mar. biol. Ass. U.K. 23: 340-341.

SCHOLL, G. 1963. Embryiologische Untersuchungen an Tanaidaceen (Heterotanais oerstendi Kroyer). Zool. Jb. Anat. 80: 3-109.

TomMASI, L.R. 1970. Observações sobre a fauna bêntica do complexo estuarino lagunar de Cananéia (SP). Bolm. Inst. Oceanogr. São Paulo 19: 43-56. 